\title{
Low Energy Beam Neutrino Nucleus Deep Inelastic Scattering at MINERvA
}

\author{
Joel MOUSSEAU* \\ $\dagger$ \\ University of Florida \\ E-mail: joelamefnal.gov
}

\begin{abstract}
Neutrinos provide a unique probe of nuclear structure as they interact weakly and couple with partons via the axial as well as the vector current. The MINERvA experiment takes advantage of Fermilab's NuMI beam line and multiple nuclear targets to study neutrino + nucleus interactions. This note presents preliminary results of MINERvA's DIS sample in the neutrino energy $\left(E_{V}\right)$ range of 5 to $50 \mathrm{GeV}$, and a mean momentum-transfer squared $\left(Q^{2}\right)$ of $4.0(\mathrm{GeV} / \mathrm{c})^{2}$. Results are presented as ratios of $\mathrm{C}, \mathrm{Fe}$ and $\mathrm{Pb}$ to $\mathrm{CH}$. The ratio of the total cross sections as a function of $E_{V}$ and differential cross sections as a function of Bjorken-x are presented.
\end{abstract}

XXIII International Workshop on Deep-Inelastic Scattering 27 April - May 12015

Dallas, Texas

* Speaker.

† On Behalf of the MINERvA Collaboration. 


\section{Introduction}

Charged lepton experiments possess a rich history of measuring $x_{b j}$ dependent nuclear effects on a variety of targets. These partonic nuclear effects are historically measured as ratios of DIS cross sections (or the structure function $F_{2}$ ) of heavy nuclei to deuterium. Decades of research have isolated four distinct types of effects in different regions of $x_{b j}$ : nuclear shadowing $\left(x_{b j}<0.1\right)$, antishadowing $\left(0.1<x_{b j}<0.3\right)$, the EMC effect $\left(0.3<x_{b j}<0.75\right)$ and Fermi motion $\left(x_{b j}>0.75\right)$.

Historically, these effects have not been well measured in neutrino physics. Attempts have been made to generate empirical fits to existing neutrino data, however these data suffer from low statistics and a lack of variety in nuclear targets. This has led to modern neutrino event generators using identical nuclear effects from charged lepton data in neutrino-nucleon scattering models. There are multiple reasons to believe this prescription is incorrect. The weak force, which solely mediates neutrino-nucleon scattering, contains vector and axial components while the electromagnetic force contains only a vector component. In addition, neutrinos experience more of the valence distribution of quarks via the structure function $x_{b j} F_{3}$.

More generally, the EMC effect has no universally accepted origin despite almost three decades of active study. A systematic study of neutrino nuclear effects in the EMC range of $x_{b j}$ has the potential to provide some insight into the physics underlying the EMC effect. To date, there are very few data of neutrino events on multiple nuclear targets. The MINERvA data, presented in this note, provide the first set of neutrino-nucleon deep inelastic scattering data on multiple nuclei in the same neutrino beam.

\section{The MINERvA Detector}

The MINERvA detector is located in the MINOS near detector cavern at Fermi National Accelerator Laboratory in Batavia, IL. MINERvA uses the NuMI facility at Fermilab as it's source of neutrinos, explained in Section 3. The cavern is located approximately 330 feet underground and the detector is located approximately $1 \mathrm{~km}$ away from the neutrino source.

The MINERvA detector is capable of measuring DIS cross sections on a variety of nuclei with very high precision. The nuclear target region contains nuclear targets of solid graphite, iron, and lead as well as a liquid water target. The fiducial masses of the various nuclear targets are in Table 1. Downstream of the target region, along the beam direction, is the tracker region composed of polystyrene $(\mathrm{CH})$ scintillator. Following the tracker are downstream electromagnetic and hadronic calorimeters. The MINOS near detector lies $2.0 \mathrm{~m}$ downstream of the MINERvA detector, and is used for measuring the energy of muons escaping MINERvA. A schematic view of the MINERvA detector with the different sub-detectors labeled is in Figure 1. 


\begin{tabular}{c|c|c|c} 
Material & Mass $(\mathrm{kg})$ & Number of Protons $\left(\times 10^{28}\right)$ & Number of Neutrons $\left(\times 10^{28}\right)$ \\
\hline $\mathrm{C}$ & 159 & 4.8 & 4.8 \\
$\mathrm{Fe}$ & 628 & 17.6 & 20.3 \\
$\mathrm{~Pb}$ & 711 & 16.9 & 25.8 \\
$\mathrm{CH}$ & 5476 & 176.0 & 1534.0 \\
\hline
\end{tabular}

Table 1: Fiducial mass of the different materials used in the DIS analysis, along with the number of nucleons per material. Table is from Ref [1].

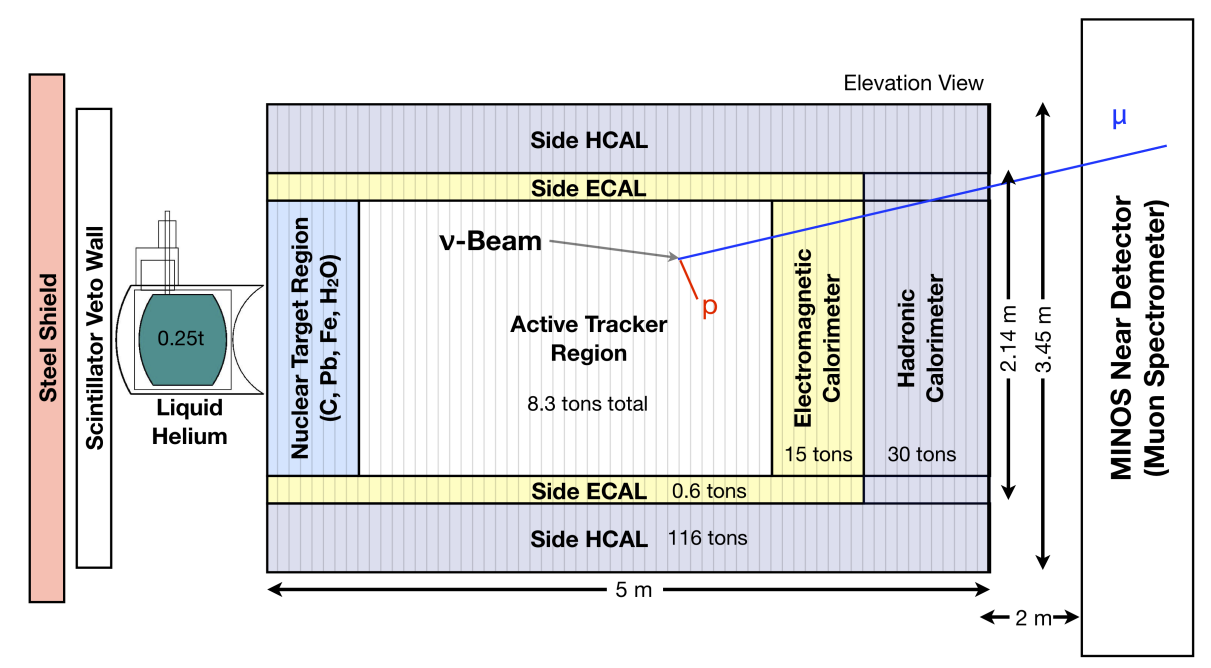

Figure 1: Schematic of the MINERvA detector, with all sub-detectors and their masses labeled. Figure is from Ref [2].

\section{NuMI Neutrino Beam}

The NuMI beam produces neutrinos by colliding a primary beam of $120 \mathrm{GeV}$ protons with a graphite target 2 interaction lengths long. Mesons created by the proton plus carbon collision are focused by a pair of magnetic horns, and allowed to decay in an evacuated decay pipe. Muons from these meson decays are stopped by rock upstream of the MINERvA detector, leaving a beam of 98 $\% v_{\mu} \mathrm{s}$.

Simulation of this hadron production, and thus hadron decay and production of neutrinos, is constrained by external data from the NA49 experiment [3]. The NA49 data however, are not able to constrain events above a neutrino energy of approximately $20 \mathrm{GeV}$. Unfortunately, roughly $50 \%$ of the MINERvA DIS event sample occurs at energies above $20 \mathrm{GeV}$. As a result, large 
uncertainties due to the model spread of the neutrino flux simulation translate to large uncertainties on the cross-section measurement.

\section{Event Reconstruction and Analysis}

Reconstructing and identifying DIS events requires a measurement of the energy of the outgoing muon $\left(E_{\mu}\right)$, the angle with respect to the neutrino beam of the muon $\left(\theta_{\mu}\right)$, the total energy of the final state hadronic shower $\left(E_{\text {had }}\right)$ and the energy of the incoming neutrino $\left(E_{v}=E_{\mu}+E_{\text {had }}\right)$. From these three variables, the invariant-mass of the hadronic system $(W)$ and the four-momentum transfer squared $\left(Q^{2}\right)$ can be measured as:

$$
\begin{array}{r}
Q^{2}=4 E_{v} E_{\mu} \sin ^{2}\left(\frac{\theta_{\mu}}{2}\right), \\
W=\sqrt{M_{N}^{2}+2 M_{N} E_{h a d}-Q^{2}},
\end{array}
$$

where $M_{N}$ is the average of the proton and neutron masses. An event is considered a DIS event if $Q^{2} \geq 1.0 \mathrm{GeV} / \mathrm{c}^{2}$ and $W \geq 1.0(\mathrm{GeV} / \mathrm{c})^{2}$. The Bjorken-x of the event is defined as:

$$
x_{b j}=\frac{Q^{2}}{2 M_{N} E_{h a d}} .
$$

The MINERvA reconstruction begins by identifying a primary muon. The primary muon is found by searching for long tracks in the MINERvA detector which match to tracks in the MINOS near detector. The start point of the primary muon track is used as an "anchor" point for a search for additional tracks in the event. When all of the secondary tracks have been found, the $(x, y, z)$ vertex of the event is re-fit iteratively using all of the tracks available and a Kalman fitter. If the event contains only one track, the start point of the primary muon is considered the vertex of the event. The MINOS matching requirement limits the angular acceptance of events in MINERvA, and a cut is made excluding muons with an exiting angle greater than $17^{\circ}$.

Once the primary muon is identified, its final momentum is measured by range in the MINOS near detector. If the muon escapes MINOS, either through the rear or sides of the detector, then the momentum is measured from its curvature in the MINOS magnetic field. Additional deposits of energy in the detector not associated with the muon track are summed to form a recoil energy. This sum is restricted to events within a -20 to +30 ns window of the event vertex. The recoil energy is corrected for passive material, containment and neutral particle content via calorimetry. This calorimetrically corrected energy sum is the $E_{\text {had }}$ of the neutrino event.

The selection of events in each nuclear target is based on the $z$ vertex of the event and include 2 scintillator planes downstream and 1 plane upstream of the target. As a consequence a subset of events reconstructed into each nuclear target truly originated in the surrounding scintillator. This background is subtracted by measuring the DIS event rate in the downstream tracker modules, and then translating the measured rate to the upstream nuclear targets. As the muon acceptance into MINOS is a strong function of the $z$ position of the vertex and the angle of the outgoing muon, each event is weighted for its MINOS acceptance via a reweighting function: 


$$
w=f\left(E_{v}, \theta_{\mu}, z\right) .
$$

$w$ is computed for a GEANT simulation of muons in the MINERvA detector.

\section{Results}

After subtracting backgrounds, the events are corrected for detector acceptance and smearing effects to form a cross section. The MINERvA flux calculation currently contains a large uncertainty $(\approx 20 \%)$ at high energies. As a result, the ratios of cross sections between the passive targets $(\mathrm{C}, \mathrm{Fe}$ and $\mathrm{Pb})$ are taken relative to the measured $\mathrm{CH}$ cross section. As all targets in MINERvA are exposed to an identical neutrino beam, uncertainties due to the flux simulation largely cancel in these ratios.

The ratios of the differential cross section with respect to Bjorken-x $\left(\frac{d \sigma}{d x}\right)$ of $\mathrm{C}, \mathrm{Fe}$ and $\mathrm{Pb}$ to $\mathrm{CH}$ are shown in Figure 2. Each plot in Figure 2 shows the data as black points with the statistical uncertainty drawn as the error bar. The red line is the prediction, and the band surrounding the prediction is the $1 \sigma$ systematic uncertainty. We observe a smaller than expected ratio in the lowest $x_{b j}$-bin $\left(0.0<x_{b j}<0.1\right)$ for the ratio of lead to scintillator. The size of this deficit relative to the simulation is suggestive of additional nuclear shadowing in lead versus scintillator.
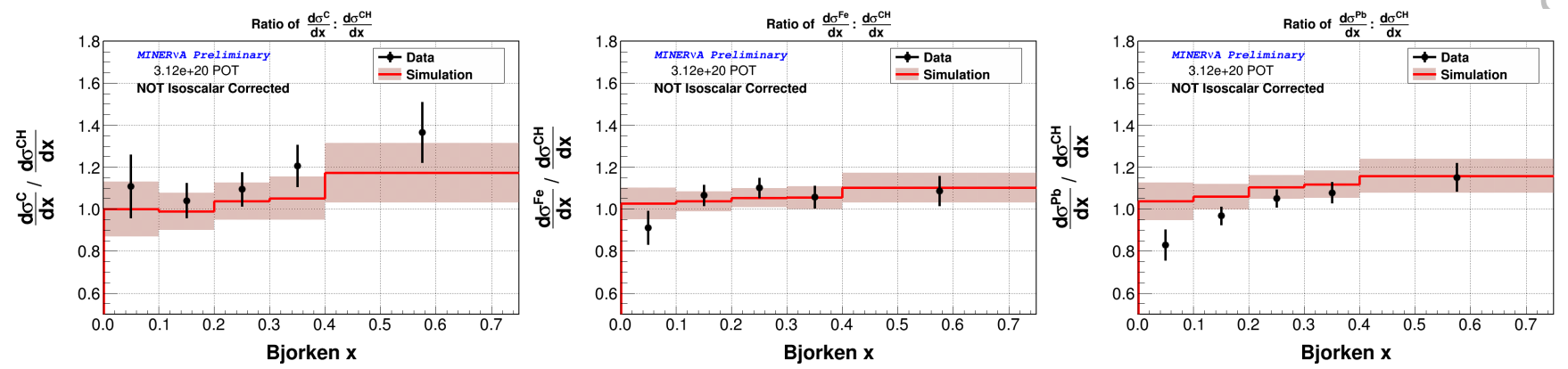

Figure 2: Ratio of the DIS $x_{b j}$ differential cross section on carbon (left), iron (center) and lead (right) divided by $\mathrm{CH}$ for data (black points) and simulation (red lines).

Each other bin of Bjorken-x shows generally good agreement between the data and prediction. This is especially true in the largest $x_{b j}$ bin, $0.4 \leq x_{b j}<0.75$, which is dominated by the EMC effect. While the data currently lack the statistical precision to differentiate between different explanations of the EMC effect, it is possible for this neutrino data to be used in developing or tuning new models of $x_{b j}$ dependent nuclear effects.

The total cross section data is compared to the simulation as a function of $E_{v}$ in Figure 3. No disagreement between the data and prediction is observed in the carbon or iron ratios. The model tends to overpredict the lead to scintillator ratio in the higher energy bins $\left(25 \leq E_{v}<40 \mathrm{GeV}\right)$. This trend appears to occur in the iron to scintillator ratio, however the data currently lack the statistical precision to resolve the potential difference. 

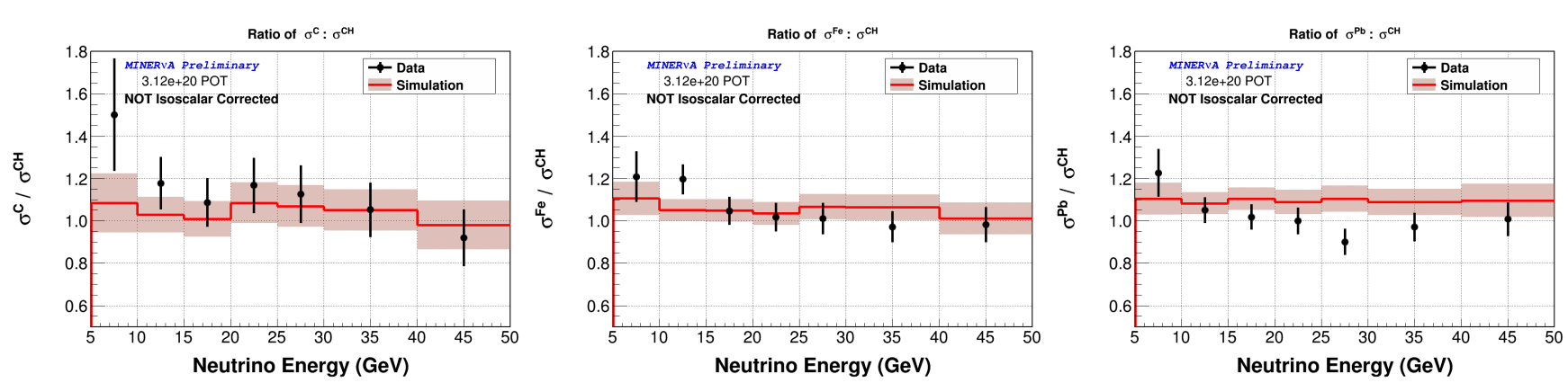

Figure 3: Ratio of the total DIS cross section as a function of neutrino energy on carbon (left), iron (center) and lead (right) divided by $\mathrm{CH}$ for data (black points) and simulation (red lines).

\section{Conclusions}

Deep inelastic scattering events are selected in the MINERvA nuclear targets based on the reconstructed $Q^{2}, W$, and $z$ position of the event. After subtracting backgrounds due to mis-identified $z$ vertices, a cross section is calculated. Due to large uncertainties on the flux calculation, the results are reported as ratios of the different passive materials in MINERvA to the scintillator cross section. The ratios show some differences between the data and simulation in the small $x_{b j}$ and large $E_{v}$ regions of lead, suggestive of additional nuclear shadowing. None of these differences are statistically significant, and there is considerable motivation for MINERvA to repeat this analysis with higher energy data currently being collected.

\section{References}

[1] B. Tice et al. [MINERvA Collaboration], "Measurement of Ratios of $v_{\mu}$ Charged-Current Cross Sections of C, Fe, and Pb to CH at Neutrino Energies 2-20 GeV," Phys. Rev. Lett. 112, 231801 (2014)

[2] L. Aliaga et al. "Design, Calibration, and Performance of the MINERvA Detector," Nucl. Instrum. Meth. 743C. 130-159, (2014).

[3] Eur. Phys. 49, 897-917 (2007). 